\title{
A Survey of Internet of Things and its Applications
}

\author{
Prof. Vivek Pandey ${ }^{1}$, Vipul Kushwaha ${ }^{1}$, Ansari Rukhsar ${ }^{1}$, Snehal Shinde ${ }^{1}$ \\ Department ofComputer Engineering, ARMIET College, Thane, India ${ }^{1}$
}

\begin{abstract}
Internet of Things (IoT) is a wide field that has a huge variety of applications. A lot of work has been done in the different fields this concept encompasses such as media, environmental monitoring, infrastructure management, manufacturing, medical and healthcare, transportation etc. This paper is a survey of the various applications of Internet of Things. This paper also focuses on the readily available platforms for the development in this field. Finally, different issues in practical applications have been discussed.
\end{abstract}

Keywords: IoT; Microcontroller; Sensor; Actuator; Wi-Fi; MQTT, Arduino YUN.

\section{INTRODUCTION}

The Internet of things (IoT) is the inter-networking of Radio-frequency identification (RFID) was popularized physical devices, vehicles (also referred to as "connected and promoted by Kevin Ashton (one of the founders of the devices" and "smart devices"), buildings, and other original Auto-ID Center) as an essential technology for items - embedded with electronics, software, sensors, IoT [9].

actuators, and network connectivity that enable these objects to collect and exchange data[1].

It has its various fields and applications such as home automation, smart materials, energy management, medical and healthcare, multimedia etc.

A broader concept of smart cities is trending nowadays. Smart cities are currently under development and are a major investment sector. [2] explains how sensors can be used to collect data from every architectural structure of a city and use it to monitor the city.

CISCO estimates that 50 billion devices would be connected to Internet by 2020 [5] and the number would increase further in a very short amount of time. Many government funded research institutes are working on creating a product that allows controlling household appliances using mobile apps, one such example is [6].

\section{HISTORY}

The concept of a network of smart devices was discussed as early as 1982, with a modified Coke machine at Carnegie Mellon University becoming the first Internetconnected appliance, [12] able to report its inventory and whether newly loaded drinks were cold.

A 1991 paper on ubiquitous computing by Mark Weiser titled "The Computer of the 21st Century" made IoT a matter of discussion among computer scientists.

Today protocols such as MQTT developed by IBM are used to communicate with sensors in a limited bandwidth.

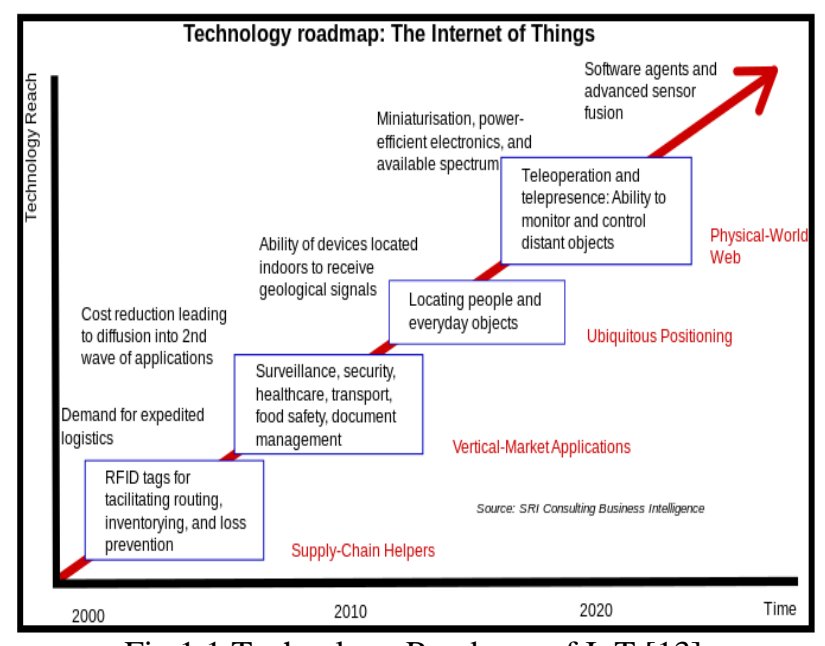

Fig.1.1 Technology Roadmap of IoT [13]

\section{III.APPLICATIONS}

1) Media

Internet of Things helps firms collect and analyze valuable user data such as behavioral patterns. This kind of data helps these firms target their specialized and advanced services to specific users thus increasing their business.

2) Manufacturing

IoT in industrial manufacturing gives rise to a new term

The concept of the Internet of things became popular in 1999, through the Auto-ID Center at MIT and related market-analysis publications.

IIoT (Industrial Internet of Things). IIoT could lead the world to the fourth industrial revolution, the so called Industry 4.0. 
Cyberphysical systems will be an interface between humans and the cyber world. It consists of 5Cs:

Connection

Conversion

Cyber

Cognition

Configuration architecture[10].

It will transform the collected data into actionable information, and eventually interfere with the physical assets to optimize processes.

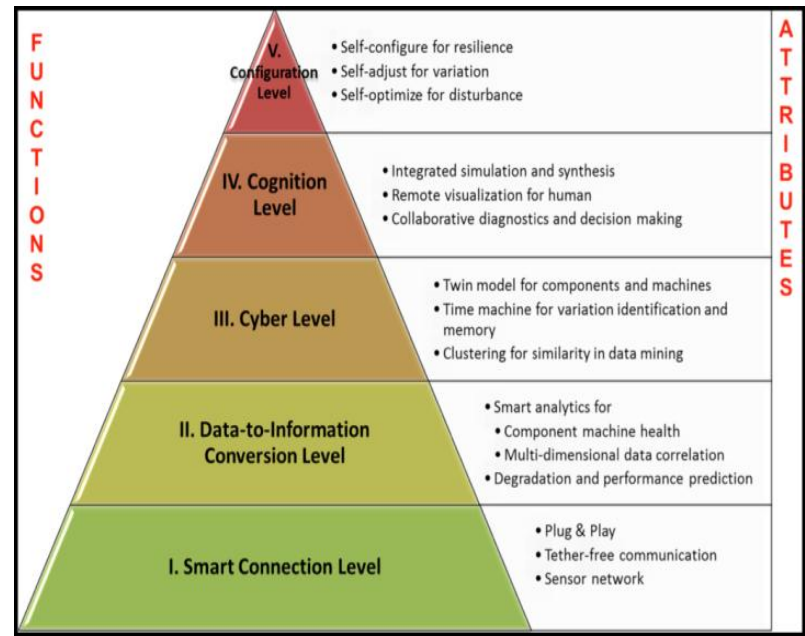

Fig. 1.2 Design architecture of cyber-physical systemsenabled manufacturing system[13]

3) Infrastructure management

Monitoring urban and rural infrastructures such as bridges, dams, highways is facilitated by the use of smart materials. Smart materials are the materials used in construction that have sensors that measure the vibrations, different forces, pressure etc. and determine whether the structure is safe for the people or not.

\section{4) Medical and healthcare}

IoT devices can be used for remote monitoring and emergency notification. An example of such a device would be smart pacemaker that notifies the concerned doctor in case of anomalies. The concept also extends to wearables such as pedometers, heart rate monitors etc.

\section{5) Energy Management}

Sensor and actuator combinations can be easily used to increase the efficiency of a system such as grids. For example, a sensor that detects light intensity can be used in combination with a light bulb to make an outdoor lamp that lights up only when it is dark enough thus saving energy as people tend to forget to turn off outdoor lights during the day.

\section{6) Building and home automation}

Home automation has been around for decades now, but due to recent developments in IoT it can be made available to a larger part of the population.

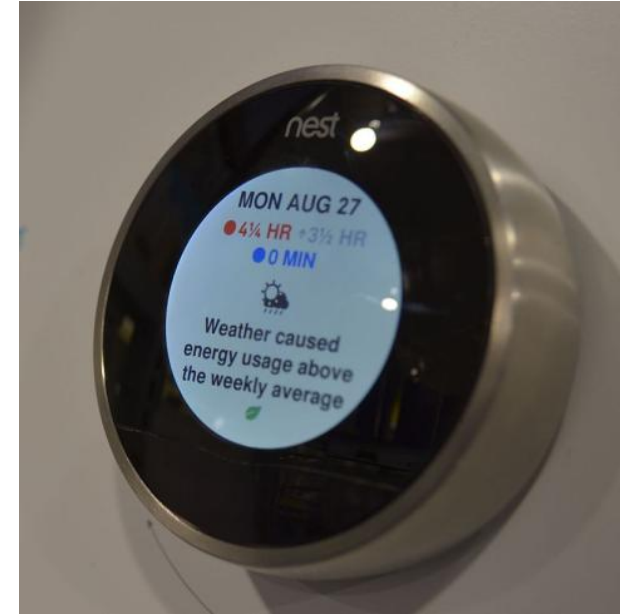

Fig. 1.3 A NEST Learning Thermostat[13]

\section{7) Transportation}

Implementing sensor based smart traffic control can help resolve traffic issues. Smart parking, electronic toll collection systems, logistic and fleet management, vehicle control, and safety and road assistance all use similar data.[11]

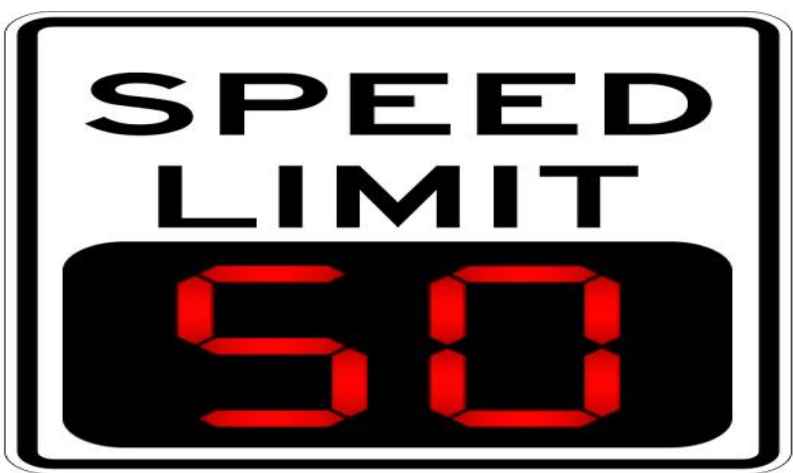

Fig. 1.4 A variable speed limit sign[13]

\section{IV.FRAMEWORKS FOR DEVELOPMENT}

Many development environments and development boards exist specifically for IoT. The most popular one is the Arduino IDE and Arduino boards. Some of the boards are well equipped with wifi capabilities such as the Arduino Yun whereas others such as Arduino UNO can be connected to the Internet using Ethernet Shield.

It is also crucial to discuss various APIs that can be used to quickly build IoT projects. Some examples are IBM Bluemix, Adafruit IO, IBM Watson, Cayenne etc. These APIs charge the developer as he expands his projects.

Protocols like MQTT are now widely used for low bandwidth communication among IoT devices and servers.

REST is a scalable architecture that allows things to communicate over Hypertext Transfer Protocol and is easily adopted for IoT applications to provide communication from a thing to a central web server. 


\section{ENABLING TECHNOLOGIES}

\section{REFERENCES}

The most trivial element in IoT is the connection of the IoT device to the Internet. These following are classified according to their operating range:

1) Short-range wireless

Bluetooth low energy (BLE)

Light-Fidelity (Li-Fi)

Near-field communication (NFC)

QR codes and barcodes

Radio-frequency identification (RFID)

Wi-Fi

ZigBee

2) Medium-range wireless

HaLow

LTE-Advanced

3) Long-range wireless

Low-power wide-area networking (LPWAN)

Very small aperture terminal (VSAT)

4) Wired

Ethernet

Multimedia over Coax Alliance (MoCA)

Power-line communication

\section{VI.ISSUES}

The major issue that IoT currently suffers from is the lack of standardization. Due to fragmentation of platforms hardware and software inconsistencies occur. There are two types of systems: open and proprietary. Open systems are difficult to standardize and proprietary systems have not yet established a majority share.

Another, issue is the threat to individual user's privacy. Hacking into IoT devices is relatively easier and can lead to disclosure of confidential data such as passwords, bank account credentials etc.

A lot of research and development will be required to make IoT systems acceptably secure.

\section{CONCLUSION AND FUTURE SCOPE}

IoT can be considered as the next step towards making everything smart. It has many applications and opportunities that keep on adding up day by day making it something that is going to stay with us for many years to come.

\section{ACKNOWLEDGMENT}

The authors express special thanks and gratitude to ARMIET College, Thane for its support in the research and development in IoT by conducting adequate amount of workshops related to this field.
[1] Brown, Eric (13 September 2016). "Who Needs the Internet of Things?". Linux.com. Retrieved 23 October 2016.A. Zanella, N. Bui, A. Castellani, L. Vangelista and M. Zorzi, "Internet of Things for Smart Cities", IEEE Internet of Things Journal, vol. 1, no. 1, pp. 22-32, 2014

[2] https://madeby.google.com/home/

[3] https://www.amazon.com/Amazon-Echo-Bluetooth-Speaker-withWiFi-Alexa/dp/B00X4WHP5E

[4] http://www.cisco.com/c/en/us/solutions/internet-ofthings/overview.html

[5] K. Mandula, R. Parupalli, C. Murty, E. Magesh and R. Lunagariya, "Mobile based Home Automation using Internet of Things(IoT)", 2015 International Conference on Control,Instrumentation, Communication and Computational Technologies (ICCICCT), 2015.

[6] Vinay sagar K N,Kusuma S M, "Home Automation Using Internet of Things", International Research Journal of Engineering and Technology (IRJET) e-ISSN: 2395 -0056 Volume: 02 Issue: 03 | June-2015

[7] Abhay Kumar, Neha Tiwari, "Energy Efficient Smart Home Automation System", International Journal of Scientific Engineering and Research (IJSER) ISSN (Online): 2347-3878 Volume 3 Issue 1, January 2015

[8] ByeongkwanKang,SunghoiPark,Tacklim Lee andSehyun Park, "loT based Monitoring System using Tri-level Context Making Model for Smart Home Services", 2015 IEEE International Conference on Consumer

[9] Magrassi, P. (2 May 2002). "Why a Universal RFID Infrastructure Would Be a Good Thing". Gartner research report G00106518.

[10] Lee, Jay; Bagheri, Behrad; Kao, Hung-An (2015). "A cyberphysical systems architecture for industry 4.0-based manufacturing systems". Manufacturing Letters. 3: 18-23.

[11] Ersue, M.; Romascanu, D.; Schoenwaelder, J.; Sehgal, A. (4 July 2014). "Management of Networks with Constrained Devices: Use Cases". IETF Internet Draft.

[12] "The "Only" Coke Machine on the Internet". Carnegie Mellon University.Retrieved 10 November 2014.

[13] https://en.wikipedia.org/wiki/Internet_of_things

\section{BIOGRAPHY}

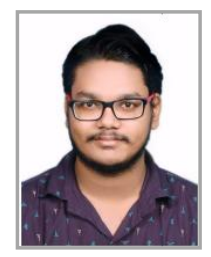

Vipul Kushwaha is a BE student at ARMIET College. Web Technologies, Networking and Embedded Systems are his areas of interest. He is currently working on an IoT project on Home Automation. 